\title{
Effect of Polymer as Cosolvent on Chemical Reactions in Solution III. Thermodynamical Cosolvent Effect of Glymes on the Reaction of Dansyl Chloride with Butylamine in Chloroform
}

\author{
Akio OKAMOTO, Tokiko HAYASHI, and Itaru MitA \\ Institute of Interdisciplinary Research, Faculty of Engineering, \\ The University of Tokyo, 4-6-1 Komaba, Meguro-ku, Tokyo 153, Japan
}

(Received November 10, 1982)

\begin{abstract}
Second-order rate constants for the reaction of 5-dimethylamino-1naphthalenesulfonyl chloride with butylamine in chloroform in the presence of glymes as oligomer cosolvents were measured by fluorometry. These cosolvents exhibit polymer effects characterized by acceleration the extent of which depended on the chainlength of the cosolvent molecules as well as the volume fraction of the cosolvent. Polymer effects by oligomer cosolvents on chemical reactions between two low molecular weight species are explained by the thermodynamics of the polymer solution.
\end{abstract}

KEY WORDS Chemical Reactions / Oligomer Cosolvents / Polymer Effect / Thermodynamics of Polymer Solution / Dansyl Chloride / Butylamine / Fluorometry / Glymes /

In a previous study, ${ }^{1}$ we reported the acceleration effects of polyoxyethylene (POE) and polystyrene (PS) on the reaction of dansyl chloride with butylamine, a reaction between two low molecular weight species, in organic solvents. These effects through the presence of polymers as cosolvents on the reaction rate were expressed in terms of the thermodynamics of polymer solution. Furthermore, we pointed out that some of the so called polymer effects can be interpreted by these thermodynamical effects. The polymer cosolvent effect is characterized by acceleration of the reaction and depends on the chainlength of the polymer molecules as well as the fraction of the polymer cosolvent. Effects from the presence of polymer cosolvents were also found in the chemical reaction between a low molecular weight species and a polymer, ${ }^{2}$ i.e., POE and PS accelerate the reaction of dansyl chloride with primary amino-ended POE in chloroform. Acceleration effects by these polymer cosolvents on reaction rate show dependence on the chainlength of the polymer molecules and fraction of the polymer cosolvent.

In this paper, we report on the effects brought about by the presence of oligomers on a chemical reaction between two low molecular weight species in solution. The effects of glymes as oligomer cosolvents on the reaction rate of dansyl chloride with butylamine in chloroform were examined. The reactions were followed by the increase in the fluorescence intensity of the reaction product. The thermodynamical effects by the oligomer cosolvent are discussed in detail in terms of dependence on the fraction of the oligomer cosolvent and chainlength of the oligomer.

\section{EXPERIMENTAL}

\section{Materials}

5-Dimethylamino-1-naphthalenesulfonyl chloride (dansyl chloride; DNSCl) was obtained from Tokyo Kasei Co. and used without further purification. Butylamine $\left(n-\mathrm{BuNH}_{2}\right.$; from Tokyo Kasei Co.) was distilled before use. The solvent used was "Dotite Spectrosol" grade chloroform (CF; from Wako Pure Chemical Industries Co.) and used as received. $\quad \alpha, \omega$-Dimethoxy oligo(oxyethylene)s (glymes; $\mathrm{CH}_{3} \mathrm{O}\left(\mathrm{C}_{2} \mathrm{H}_{4} \mathrm{O}\right)_{n} \mathrm{CH}_{3}$ ) were monoglyme 
$(n=1)$, diglyme $(n=2)$, triglyme $(n=3)$, and tetraglyme $(n=4)$. These glymes were obtained from Tokyo Kasei Co. and purified. A typical example of purification is given below. An aqueous solution of $1 N \mathrm{NaOH}(10 \mathrm{ml})$ was added to tetraglyme $(100 \mathrm{ml})$. The resulting mixture was stirred overnight at room temperature. The transparent supernatant solution $(c a .90 \mathrm{ml})$ was then distilled under reduced pressure. To ensure sufficient purity of the glyme for subsequent kinetic studies, DNSCl was dissolved in the glyme and the mixture was allowed to stand at $60^{\circ} \mathrm{C}$ overnight. No side reactions were detected at all. The glyme was completely nonfluorescent. $N$-Butyl-5-dimethylamino1-naphthalenesulfonamide (DNSBA), the reaction product of $\mathrm{DNSCl}$ with $n-\mathrm{BuNH}_{2}$, was prepared and purified separately to examine its fluorescence prior to kinetic measurements. ${ }^{3}$

\section{Measurements}

The reaction product of $\mathrm{DNSCl}$ with $n-\mathrm{BuNH}_{2}$ is fluorescent but not the reactants. The reaction rates can be followed by fluorescence measurements ${ }^{3}$ and were performed with a JASCO FP-550 spectrofluorometer without degassing. The fluorescence intensity of DNSBA in chloroform decreased by a few percent by the addition of $n-\mathrm{BuNH}_{2}$ up to about ten fold excess to DNSBA in molality, but remained constant on further addition of $n-\mathrm{BuNH}_{2}$. In the following kinetic studies, the fluorescence intensity of the reaction product was corrected for the effects of excess $n-\mathrm{BuNH}_{2}$. The fluorescence intensity of DNSBA, however, was not altered by the addition of glymes.

\section{RESULTS AND DISCUSSION}

\section{Thermodynamics of acceleration effects by oligomer cosolvent}

Acceleration effects through the oligomer cosolvent on chemical (not diffusion controlled but activation controlled) reactions can be expressed by the change in reactant activities. These effects are essentially the same as the polymer effects resulting from the polymer cosolvent, on consideration of the thermodynamics of the polymer solution. ${ }^{1,2}$ According to the Brönsted-Bjerrum theory, ${ }^{4}$ the second-order rate constants $k_{2}$ and $k_{2}^{0}$ for the chemical reaction

$$
\mathrm{A}+\mathrm{B} \rightleftharpoons \mathrm{X} \longrightarrow \mathrm{P}
$$

are related to the activity coefficients of the reactants $A$ and $B$, and the activated complex $X$, in the form

$$
k_{2} / k_{2}^{0}=\gamma_{\mathrm{A}} \cdot \gamma_{\mathrm{B}} / \gamma_{\mathbf{X}}
$$

where $k_{2}^{0}$ is the second-order rate constant of the reaction in the ideal solution, $k_{2}$, that in a given solution, and $\gamma_{A}, \gamma_{B}$, and $\gamma_{X}$, the activity coefficients of $\mathrm{A}, \mathrm{B}$, and $\mathrm{X}$, respectively.

The ratio of the second-order rate constants for the chemical reaction in the presence of an oligomer cosolvent with the degree of polymerization $m$ and with the degree of polymerization $n$ can be obtained as a function of activities from eq 2 to be

$$
\frac{k_{2}^{m}}{k_{2}^{n}}=\frac{a_{\mathrm{A}}^{m} \cdot a_{\mathrm{B}}^{m}}{a_{\mathbf{X}}^{m}} \cdot \frac{a_{\mathbf{X}}^{n}}{a_{\mathrm{A}}^{n} \cdot a_{\mathbf{B}}^{n}}
$$

where $a_{\mathrm{A}}^{m}, a_{\mathrm{B}}^{m}$, and $a_{\mathrm{X}}^{m}$ are the activities of $\mathrm{A}, \mathrm{B}$, and $X$, respectively, for the reaction system in the presence of the oligomer cosolvent with the degree of polymerization $m$, etc.

As indicated above, the activity $a_{i}$ for the $i$ th constituent in a polycomponent system is given as $^{5,6}$

$$
\begin{aligned}
\ln a_{i}= & \ln v_{i}+\left(1-v_{i}\right)-\sum_{j \neq i}\left(\frac{m_{i}}{m_{j}}\right) v_{j} \\
& +\left(1-v_{i}\right) \sum_{j \neq i} \chi_{i j} v_{j}-\sum_{\substack{j, k \neq i \\
j<k}}\left(\frac{m_{i}}{m_{j}}\right) \chi_{j k} v_{j} v_{k}
\end{aligned}
$$

where $m_{i}$ is the degree of polymerization of the $i$ th component and is regarded as the ratio of the molar volume to the common unit molar volume and $\chi_{i j}$ a pair-interaction parameter between components $i$ and $j$.

The value of $\chi_{i j}$ is usually regarded as proportional to $m_{i}$, ${ }^{6}$ but its molecular weight dependence is not simple. ${ }^{7}$ According to Koningsveld et al., $\chi_{i j}$ may be written as a first approximation as

$$
\chi_{i j} / m_{i}=\chi_{i j}^{\prime}+\chi_{i j}^{\prime \prime} / m_{i}
$$

where $\chi_{i j} / m_{i}$ is an alternative expression of the pairinteraction parameter and $\chi_{i j}^{\prime}$ and $\chi_{i j}^{\prime \prime}$ are independent of $m_{i}$. It should be noted that for polymers of high molecular weight $\left(m_{i} \rightarrow \infty\right), \chi_{i j}^{\prime \prime} / m_{i}$ is neglected and $\chi_{i j}^{\prime}$ (or according to Flory's definition $\left.m_{i} \chi_{i j}^{\prime}\right)$ is regarded as the pair-interaction parameter. In this particular case for oligomers, however, the 
term $\chi_{i j}^{\prime \prime}$ functions efficiently in the acceleration effects by oligomer cosolvent as shown below.

From eq 3 to 5, we have

$$
\ln \left(\frac{k_{2}^{m}}{k_{2}^{n}}\right)=\left(\frac{1}{n}-\frac{1}{m}\right)\left(2 \chi_{\mathrm{CX}}^{\prime \prime}-\chi_{\mathrm{CA}}^{\prime \prime}-\chi_{\mathrm{CB}}^{\prime \prime}\right) v_{\mathrm{C}}
$$

Equation 6 indicates that acceleration effects by oligomer cosolvent can be expressed by the product of three terms: (i) the term for chainlength dependence, (ii) that for the difference in the pairinteraction parameters $\chi_{i j}^{\prime \prime}$, and (iii) the volume fraction of the oligomer cosolvent $v_{\mathrm{c}}$. This is the thermodynamical expression for the acceleration effects by oligomer cosolvent on the chemical reactions between two low molecular weight species. To demonstrate the validity of eq 6 with respect to terms $v_{\mathrm{C}}$ and $(1 / n-1 / m)$, the reaction between the two low molecular weight species in the presence of an oligomer cosolvent was investigated.

\section{Acceleration effects through glymes as oligomer cosolvents}

The reactions of $\mathrm{DNSCl}$ with $n-\mathrm{BuNH}_{2}$ in the presence of glymes as oligomer cosolvents were investigated by fluorometry. The second-order rate constants for the reaction were calculated from the observed pseudo first-order rate constants for a system with excess amine. Figure 1 shows the cosolvent effects on the second-order rate constants of the reaction of $\mathrm{DNSCl}$ with $n-\mathrm{BuNH}_{2}$ in chloroform at $40^{\circ} \mathrm{C}$ by cosolvent oligomers or glymes: monoglyme, diglyme, triglyme, and tetraglyme. Figure 1 indicates that acceleration effects by glymes can be classified according to two regions: a rapid acceleration region with a volume fraction of glymes up to $c a .20 \%$ and a gentle acceleration region thereafter. In our previous study, ${ }^{1}$ it was found that the addition of diethoxyethane (DEE) accelerated the reaction rate of $\mathrm{DNSCl}$ with $n$ $\mathrm{BuNH}_{2}$ in CF. Acceleration by DEE was attributed to the coordination of two oxygen atoms in the molecule with the transition state in the reaction. Acceleration due to this coordination seemed saturated at $c a .20 \mathrm{vol} \%$ of DEE. Further, the addition of DEE beyond ca. $20 \mathrm{vol} \%$ reduced the rate of reaction. In the present study, acceleration by the addition of glymes up to $c a$. $20 \mathrm{vol} \%$ may also be attributed to the coordination of oxygen atoms in the glymes with the transition state in the reaction. In constrast to DEE, however, the addition of

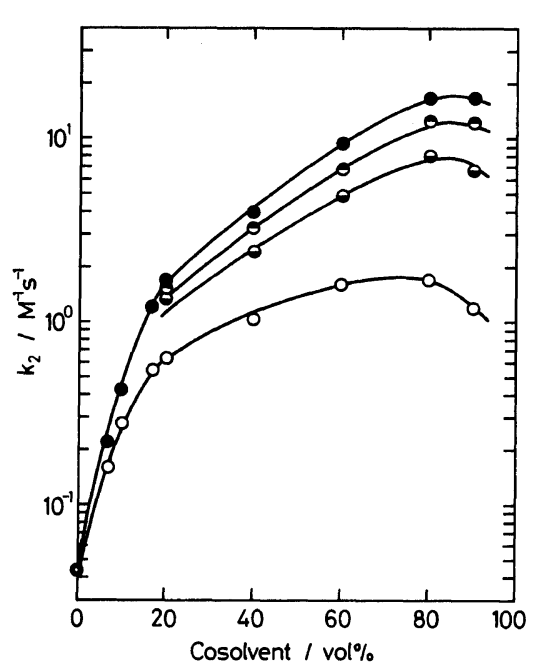

Figure 1. Plots of $k_{2} v s . v_{\mathrm{C}}$ for the reaction of DNSCl with $n-\mathrm{BuNH}_{2}$ in $\mathrm{CF}$ at $40^{\circ} \mathrm{C}$ with cosolvents: monoglyme $(O)$, diglyme $(\Theta)$, triglyme $(\Theta)$, and tetraglyme (O).

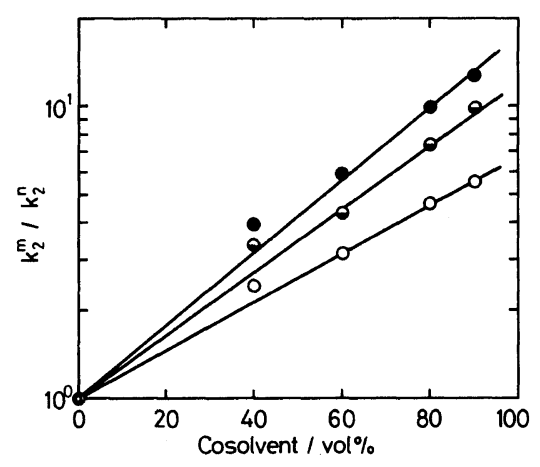

Figure 2. Plots of $\ln \left(k_{2}^{m} / k_{2}^{n}\right)$ vs. $v_{\mathrm{C}}$ for the reaction of DNSCl with $n-\mathrm{BuNH}_{2}$ in $\mathrm{CF}$ at $40^{\circ} \mathrm{C}$ with cosolvent glymes: diglyme/monoglyme $(\mathrm{O})$, triglyme/monoglyme $(\ominus)$, and tetraglyme/monoglyme (O).

monoglyme beyond $c a .20 \mathrm{vol} \%$ did not reduce the reaction rate but gradually accelerated it. This discrepancy may be explained by the difference in the solvent power of DEE and monoglyme, viz., the solubility of organic compounds in monoglyme is generally better than in DEE. Figure 1 shows that acceleration effects through glymes of longer chainlength are always more than those by glymes of shorter chainlength. The chainlength dependence of acceleration by glymes becomes more evident with increasing volume fraction of the 


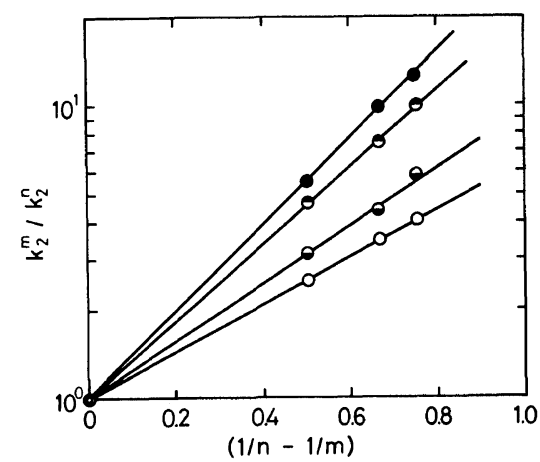

Figure 3. Plots of $\ln \left(k_{2}^{m} / k_{2}^{n}\right)$ vs. $(1 / n-1 / m)$ for the reaction of DNSCl with $n-\mathrm{BuNH}_{2}$ in CF at $40^{\circ} \mathrm{C}$ with cosolvent glymes of different $v_{\mathrm{C}}: v_{\mathrm{C}}=40(\bigcirc), 60(\Theta), 80$ $(\ominus)$, and $90(\odot) \mathrm{vol} \%$.

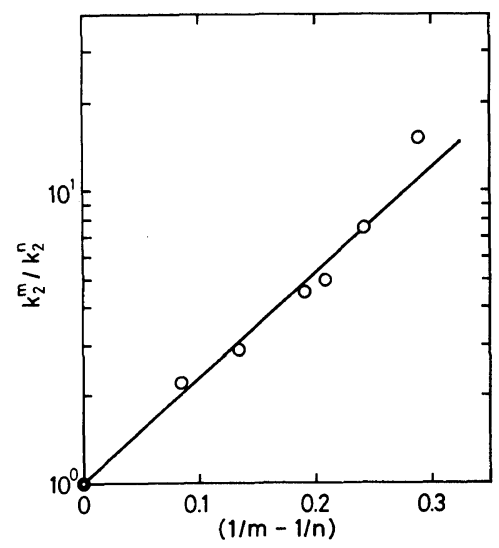

Figure 4. Plot of $\ln \left(k_{2}^{m} / k_{2}^{n}\right) v s$. $(1 / n-1 / m)$ for the butylaminolysis of $p$-nitrophenyl acetate in chlorobenzene at $25^{\circ} \mathrm{C}$ with glymes (from ref 8 ).

oligomer cosolvent. This acceleration has the characteristics of the oligomer cosolvent as explained by the thermodynamics of the polymer solution in the preceding section (eq 6).

Dependence of acceleration on glyme volume fraction

Equation 6 shows the dependence of acceleration effects from the oligomer cosolvent on the volume fraction of the cosolvent for the reaction of DNSCl with $n-\mathrm{BuNH}_{2}$ in $\mathrm{CF}$ in the presence of glymes. Figure 2 shows the $\ln \left(k_{2}^{m} / k_{2}^{m}\right)-v_{\mathrm{C}}$ plots from the data in Figure 1, with monoglyme as a reference. The plots are linear in accordance with the relation expressed by eq 6 . From the slope of lines in Figure 2 and values of $(1 / n-1 / m)$, we obtained values of $\chi_{i j}^{\prime \prime}: 3.8$ for diglyme/monoglyme, 3.9 for triglyme/ monoglyme, and 3.9 for tetraglyme/monoglyme. These values are in fair agreement with each other and are compatible with the values 4.7 and 5.3 obtained from the polymer cosolvent effects by POE on the same reaction. ${ }^{1}$ The significance of these values cannot be commented on in detail since $\chi_{i j}^{\prime \prime}$, the pair-interaction parameter, between the polymer or oligomer cosolvent and the activated complex cannot be estimated. The validity of eq 6 with respect to the term $v_{\mathrm{C}}$, however, is shown by these and previous results.

\section{Chainlength dependence of acceleration effects by glymes}

Equation 6 shows the dependence of acceleration on chainlength for the reaction of $\mathrm{DNSCl}$ with $n$ $\mathrm{BuNH}_{2}$ in $\mathrm{CF}$ in the presence of glymes. Figure 3 shows the $\ln \left(k_{2}^{m} / k_{2}^{n}\right)-(1 / n-1 / m)$, plots from the data in Fig. 1, with monoglyme as a reference monomer $(n=1)$. The lines are all linear in accordance with the relation expressed by eq 6 . From the slopes of the lines in Figure 3 and values of $v_{\mathrm{C}}$, we obtained consistent values for the term $\chi_{i j}^{\prime \prime}$ $(4.0 \pm 0.2)$.

Acceleration effects by glymes on ester aminolysis in nonpolar media have been reported by Hogan et $a l .^{8}$ These effect on the aminolysis of $p$-nitrophenyl acetate by butylamine in chlorobenzene increases with the number of oxygens in the glymes. These authors maintain that this is due simply to the fact that the complexation constants of glymes complexed to the activated complex of the reaction increase with the number of oxygen atoms per glyme molecule. But in contrast to this, we consider that acceleration effects on ester aminolysis may be explained as arising from the oligomer cosolvent effects dealt with here. The values of $\ln \left(k_{2}^{m} / k_{2}^{n}\right)$ calculated from the data of Hogan et al $^{8}$ are plotted against $(1 / n-1 / m)$ with diglyme as a reference $(n=2)$ in Figure 4. The plot gives a linear line, indicating that acceleration effects on ester aminolysis can be explained on the basis of oligomer cosolvent effects (eq 6).

In conclusion, oligomer cosolvent effects on chemical reactions were demonstrated for the reaction of $\mathrm{DNSCl}$ with $n$ - $\mathrm{BuNH}_{2}$ in $\mathrm{CF}$ with glymes as oligomer cosolvents. These effects are essentially the same as those of the polymer cosolvent as explained on the basis of the thermodynamics of the 
polymer solution. The relation expressed by eq 6 was verified with respect to the term $v_{\mathrm{C}}$ as well as the term $(1 / n-1 / m)$ as shown in Figures 2 to 4.

\section{REFERENCES}

1. A. Okamoto, A. Hayashi, K. Uchiyama, and I. Mita, Eur. Polym. J., 19, 399 (1983).

2. A. Okamoto, A. Hayashi, and I. Mita, Eur. Polym. J., 19, 405 (1983).
3. A. Okamoto, K. Uchiyama, and I. Mita, Bull. Chem. Soc. Jpn., 55, 3068 (1982).

4. J. N. Brönsted, Z. Phys. Chem., 115, 337 (1925); N. Bjerrum, ibid., 118 (1925).

5. P. J. Flory, "Principle of Polymer Chemistry," Cornell Univ. Press, Ithaca, New York, 1953, p 549.

6. I. Mita, J. Macromol. Sci., Chem., A8, 1273 (1974).

7. R. Koningsveld and L. A. Kleintjens, Macromolecules, 4, 637 (1971).

8. J. C. Hogan and R. D. Gandour, J. Am. Chem. Soc., 102, 2865 (1980). 